\title{
Morphine pre- and post-conditioning exacerbates apoptosis in rat hippocampus cells in a model of homocysteine-induced oxidative stress
}

\author{
AHMAD KARKHAH ${ }^{1,2}$, RAMIN ATAEE ${ }^{3}$ and AMIN ATAIE ${ }^{1}$ \\ ${ }^{1}$ Neuroscience Research Center, Babol University of Medical Sciences; ${ }^{2}$ Student Research Committee, \\ School of Medicine, Babol University of Medical Sciences, Babol; ${ }^{3}$ Pharmaceutical Sciences Research Center, \\ Department of Pharmacology and Toxicology, Mazandaran University of Medical Sciences, Sari, Mazandaran, Iran
}

Received February 6, 2017; Accepted June 23, 2017

DOI: $10.3892 /$ br.2017.962

\begin{abstract}
Recent investigations indicated that morphine has protective effects in different ischemia/reperfusion models and may protect against neuronal cell death, while other evidence showed that morphine induces apoptosis in neurons. Therefore, the current study was conducted to investigate preand post-conditioning effects of morphine on hippocampal cell apoptosis in a rat model of homocysteine (Hcy)-induced oxidative stress. In the present study, $0.5 \mu \mathrm{mol} / \mu 1 \mathrm{Hcy}$ was injected into bilateral intrahipocampal in the rat brain and morphine at a therapeutic dose of $10 \mathrm{mg} / \mathrm{kg}$ was injected intraperitoneally 5 days before and after Hcy injection in rats. The left and right rat hippocampus were removed for biochemical and histopathological analysis. In addition, hippocampal cell apoptosis was assayed by the TUNEL kit. Our results indicated that malondialdehyde (MDA) and superoxide anion (SOA) levels in the Hcy group were increased significantly compared to the control group $(\mathrm{P}<0.001)$. In addition, morphine pre- and post-treatment increased the MDA and SOA levels significantly in rat hippocampus compared with other groups $(\mathrm{P}<0.001)$. It was found that Hcy alone induced apoptosis in hippocampus cells and significantly increased the number of TUNEL-positive cells in rat hippocampus compared to the other group $(\mathrm{P}<0.001)$. Notably, our results indicated that pre- and post-treatment by morphine increased apoptosis in hippocampus cells compared with the other group $(\mathrm{P}<0.001)$. In conclusion, morphine neuroprotection and neurotoxicity needs to be further investigated to determine morphine side-effects in medical applications and to identify new targets for potential therapies.
\end{abstract}

Correspondence to: Dr Amin Ataie, Neuroscience Research Center, Babol University of Medical Sciences, Ganj Afrooz Avenue, Babol, Mazandaran, Iran

E-mail: ahmadkarkhah@ymail.com

Key words: homocysteine, morphine, TUNEL, hippocampus, rat, apoptosis, oxidative stress

\section{Introduction}

Morphine has been a widely used medicine in clinics for a long time as an effective treatment for diarrhea and pain. Several lines of evidence demonstrated that morphine is neuroprotective. Involvement of opioids, currently used in severe pain treatments, and in neurodegeneration/neuroprotection mechanisms, is an important field of study. Previous findings have shown that opioid receptors are involved in neuroprotection $(1,2)$.

In rat neonatal hypoxia-ischemia model, morphine application immediately after hypoxia decreased the infarct volume in the brain (1). In zebra fish embryos, morphine at certain concentrations, enhances neuron proliferation and the number of certain neuronal populations and also protects against glutamate damage in motor neurons and Pax-6-positive neurons in vivo (3). In addition, morphine is protective against microglia-mediated lipopolysaccharide- or 1-methyl-4-phenylpyridinium-induced dopaminergic neurotoxicity in rat primary mesencephalic neuron/glia cultures (4). In rat neuronal/glial cultures, morphine was reported to prevent cell death induced by HIV envelope glycoprotein gp120IIIB or BaL (5). Exogenous morphine pre-incubation improves the population of spike amplitudes of evoked field potentials, indicating that pre-conditioning with morphine may be neuroprotective (6). In addition, morphine pre-conditioning induces opioid receptor-dependent neuroprotection against ischemia in rats and reduces ischemia-induced cell death in the CA1 regions of hippocampal slices (2). In mouse hippocampus slices with oxygen-glucose deprivation, morphine pre-conditioning improves the neuronal cell survival rate through protein kinase C (7). However, many investigations showed that morphine could protect against neuronal cell death, there are several lines of evidence suggesting that morphine induces apoptosis in neurons. The long-term effect of morphine on cerebral neurons indicates that use of opioids may induce the structural alteration of neurons. On the other hand, morphine is associated with increased metabolism and oxidative damage to cells by reducing the intracellular dopamine level which leads to neuronal death (8). Previous findings have also shown that repeated application of morphine is neurotoxic for the neuronal system and spinal cord and 
triggers apoptosis (9). In addition, it was reported that morphine may cause neuronal apoptosis by altering the expression of Fas, Bcl-2 and caspase-3 (10). Therefore, because improved understanding of morphine neuroprotection and neurotoxicity may be useful to control morphine side-effects in medical applications and to identify new targets for potential therapies and prevention strategies to opioid addiction, the present study was conducted to investigate the pre- and post-conditioning effects of morphine on hippocampal cell apoptosis in a rat model of homocysteine (Hcy)-induced oxidative stress.

\section{Materials and methods}

Drugs and biochemical reagents. D-L-homocysteine, morphine, butylhydroxytoluene (BHT), 2-thiobarbituric acid (TBA), 1.1.3.3-tetramethoxypropan (99\%), nitro blue diformazan (NBD), nitro blue tetrazolium (NBT), trichloroacetic acid (TCA), butanol and ethyloleate, hematoxylin, eosin, sodium pentobarbital (USP), absolute ethanol, xylene and formaldehyde were obtained from Sigma-Aldrich, Darmstadt, Germany. Ketamine and xylazine were also purchased from Alfasan Co. (Woerden, The Netherlands). Hcy powder was dissolved in hydrochloric acid (1 M) and dilution was performed using PBS (Sigma-Aldrich). The regulation of solution $\mathrm{pH}$ at 7.4 was carried out by the addition of $0.1 \mathrm{M} \mathrm{NaOH}$. Hcy solutions were freshly prepared at a concentration of $0.5 \mu \mathrm{mol} / \mu 1$. Morphine was dissolved in distilled water at a concentration of $10 \mathrm{mg} / \mathrm{ml}$.

Animals. Twenty-one adult male Wistar rats weighing between 220 and $250 \mathrm{~g}$ were taken from the animal house. The animals were kept under controlled temperature, humidity, and lighting (12 h light/dark cycle) conditions and had free access to food and water. All experiments were performed according to the National Institutes of Health Guidelines and were approved by the Ethics Committee of Babol University of Medical Sciences (Babol, Iran). Seven rats in the sham group were injected with PBS intrahippocampally. In the Hcy group, $1 \mu \mathrm{l} \mathrm{Hcy}(0.5 \mu \mathrm{mol} / \mu \mathrm{l})$ was injected intrahippocampally and saline was injected (i.p.) 5 days before and after Hcy injection. In the Hcy-morphine group, morphine (10 mg/kg) was injected (i.p.) 5 days before and after Hcy injection.

Intrahippocampus injection (stereotaxic surgery). After anaesthetization with Ketamine-Xylosin (10 mg/kg i.p.), the rats were placed in a stereotaxic frame and the skull of the rats was orientated according to Paxinos and Watson stereotaxic atlas (11). After a sagittal incision, the bregma suture was located and holes were drilled with an electrical drill at the following co-ordinates; $3.3 \mathrm{~mm}$ posterior to bregma, $2.6 \mathrm{~mm}$ lateral to the sagittal suture and $3.6 \mathrm{~mm}$ ventral. Care was taken not to damage the meninges. A Hamilton syringe with a cannula of a diameter of $0.3 \mathrm{~mm}$ was used to inject $1 \mu \mathrm{l}$ of $0.5 \mu \mathrm{mol} / \mu \mathrm{l} \mathrm{Hcy}$ solution or its vehicle (PBS). The injection was carried out in the left and right dorsal hippocampus at a rate of $1 \mu \mathrm{l}$ per $2 \mathrm{~min}$. The cannula was left in situ for a further 5 min following Hcy injection to allow passive diffusion from the cannula tip and to minimize spread into the injection tract. The cannula was then slowly removed and the scalp was then closed with sutures. The animals were kept warm until recovery from the anesthesia.

Hippocampus removal. Five days after Hcy injection, the rats were sacrificed and the brain was exposed by making an incision through the bone on either side of the parietal suture, from the foramen magnum to near the orbit. The calvarium was removed, exposing the brain; the left and right hippocampus were removed carefully, immediately inserted in PBS solution $(0.1 \mathrm{M})$ and stored at $-70^{\circ} \mathrm{C}$ for the use in biochemical or histopathological analysis.

Hippocampus histopathological analysis. Hippocampal tissues were removed and fixed in $10 \%$ neutral-buffered formaldehyde for $24 \mathrm{~h}$, embedded in paraffin and cut into 3-4 $\mu \mathrm{m}$ sections by a microtome (Leica SM2000R; Leica Biosystems, Nussloch, Germany). The tissue sections were deparaffinised in xylene. The slides were stained with hematoxylin and eosin (H\&E) according to the procedure in Wilson and Gamble (2002), and viewed under a light microscope (Labomed, Los Angeles, CA, USA) for the structure and morphology of cells. Microscopic images were obtained by a CCD camera and Digipro software.

Assay of malondialdehyde (MDA) in hippocampal homogenate. On the day of experiment, left and right hippocampus of rat were weighed (estimate $0.7 \mathrm{~g}$ ), and homogenized (10\% w/v) in 0.1 M PBS with Polytron homogenizer at $\mathrm{pH}$ 7.4. Homogenates were used immediately for detection of the biomarkers of lipid peroxidation. Lipid peroxidation was determined according to the modified method (11). Hippocampal homogenates $(1 \mathrm{ml})$ were incubated at $37^{\circ} \mathrm{C}$ in an oscillating water bath for $1 \mathrm{~h}$. At the end of the incubation period, $0.5 \mathrm{ml}$ of BHT $(0.5 \mathrm{mg} / \mathrm{ml}$ in absolute ethanol) and $1 \mathrm{ml}$ of TCA (25\%) were added. The tubes were sealed and heated for $10 \mathrm{~min}$ in a boiling water bath to release MDA (the end product of lipid peroxidation) from proteins. To avoid adsorption of MDA to insoluble proteins, the samples were cooled to $4^{\circ} \mathrm{C}$ and centrifuged at $2,000 \mathrm{x} \mathrm{g}$ for $20 \mathrm{~min}$. Following centrifugation, $2 \mathrm{ml}$ of the protein-free supernatant was removed from each tube and $0.5 \mathrm{ml}$ of TBA $(0.33 \%)$ was added to this fraction. All the tubes were heated for $1 \mathrm{~h}$ at $95^{\circ} \mathrm{C}$ in a water bath. After cooling, the TBA-MDA complexes were extracted with $2 \mathrm{ml}$ butanol. The light absorbance was red at $532 \mathrm{~nm}$ on UV/visible spectrophotometer and MDA levels were determined from standard curve generated from 1,1,1,3 tetramethoxy propan. The results were represented as $\mathrm{nmol} / \mathrm{mg}$ wet tissue.

Assay of superoxide anion (SOA) in hippocampal homogenate. For detection of SOA the assay procedure was a modification of the method described by Das et al (12). Hippocampal homogenate $(1 \mathrm{ml})$ was incubated with $0.4 \mathrm{ml}$ of NBT $(0.1 \%)$ in an oscillating water bath for $1 \mathrm{~h}$ at $37^{\circ} \mathrm{C}$. Termination of the assay and extraction of the reduced NBT was carried out by centrifuging the samples for $10 \mathrm{~min}$ at 2,000 $\mathrm{x}$ g followed by resuspension of the pellets with $2 \mathrm{ml}$ of glacial acetic acid. The absorbance was measured at $560 \mathrm{~nm}$ on a spectrophotometer and converted to micromoles of Diformazan using a standard curve generated from NBD. The results were represented as $\mu \mathrm{mol} / \mathrm{mg}$ wet tissue. 

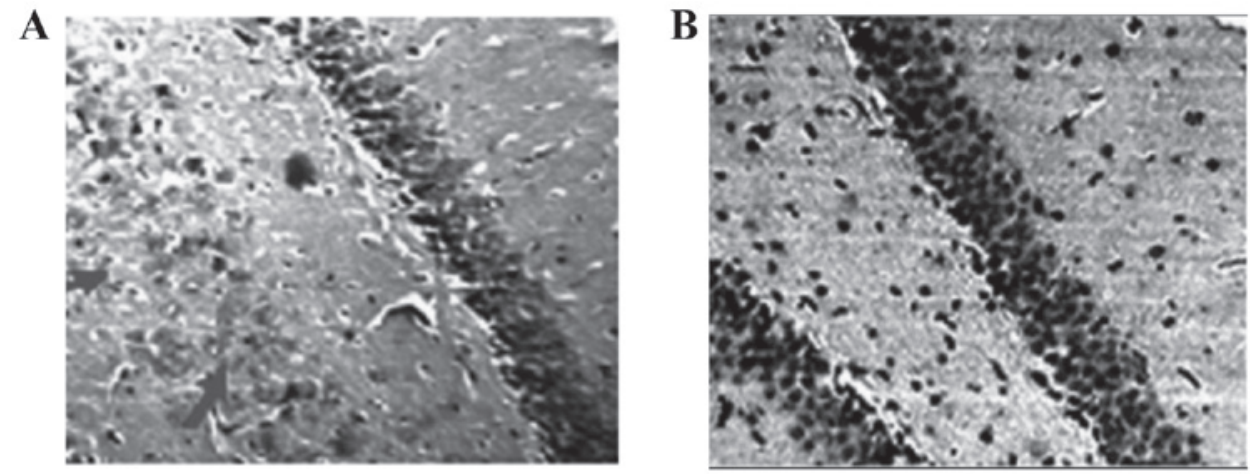

Figure 1. Images were taken from the rat hippocampal sections stained with hematoxylin and eosin; magnification, x40. (A) DG cells of Hcy group, (B) DG cells of control group. Significant changes in morphology (cell structure) were observed between groups and the cell count per $\mathrm{mm}^{2}$ (cell density of DG layer) in the Hcy group was decreased significantly in comparison with the control group $(\mathrm{P}<0.001)$. DG, dentate gyrus; Hcy, homocysteine.

Determination of hippocampus apoptosis. A terminal deoxy nucleotidyl transferase dUTP nick-end labeling (TUNEL) assay was used to assess hippocampus apoptosis with an apoptosis detection kit (Boster Biological Technology, Ltd., Wuhan, China) according to the manufacturer's instructions. For each slide, 10 fields were randomly chosen, with TUNEL-positive cells showing brown staining within the nucleus of apoptotic cells. Measurement of the apoptosis level was carried out on formalin-fixed, paraffin-embedded sections using an In situ Direct Fragmentation (TUNEL) Assay kit according to the manufacturer's instructions. First, the slides were fixed by adding $1 \%(\mathrm{w} / \mathrm{v})$ paraformaldehyde in PBS and placed on ice for $15 \mathrm{~min}$. After washing slides with PBS, ice-cold 70\% (v/v) ethanol was added to the slides after which they were allowed to stand for a minimum of $30 \mathrm{~min}$ in the freezer. Following the removal of ethanol, the slides were washed with washing buffer one more time. The slides were incubated in the prepared staining solution for $60 \mathrm{~min}$ at $37^{\circ} \mathrm{C}$. Subsequently, rinse buffer was added to each slide for $5 \mathrm{~min}$. The rinsing step was repeated one more time. Propidium iodide/RNase A solution was added to the slides and incubated in the dark for $30 \mathrm{~min}$ at room temperature. At the end of the experiments, apoptosis analysis was carried out using a fluorescence microscopy Ex/Em wavelength $(\mathrm{Ex} / \mathrm{Em}=495 / 519 \mathrm{~nm})$.

Statistical analysis. The apoptosis levels of sham, Hcy, and morphine-Hcy groups were determined. The percentage of cell death was measured separately. Data are presented as means \pm standard error of the mean (SEM) and analyzed by one-way analysis of variance (ANOVA). The data of biochemical studies were expressed as means \pm SEM and analyzed using one-way ANOVA. $\mathrm{P}<0.05$ was considered statistically significant.

\section{Results}

Histopathological analysis and validation of model. The results showed that there were significant differences in the morphology and structure of hippocampus cells between experimental groups. On the other hand, our findings indicated that the cell density of dentate gyrus (cell count per $\mathrm{mm}^{2}$ ) in the Hcy group was significantly $(\mathrm{P}<0.001)$ lower than that of the control group (Fig. 1).

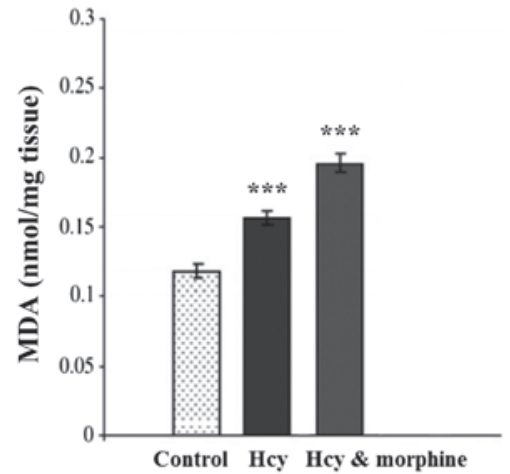

Figure 2. Effects of morphine $(10 \mathrm{mg} / \mathrm{kg})$ on MDA concentration in Hcy $(0.5 \mu \mathrm{mol} / \mu \mathrm{l})$-treated rats. Morphine pre- and post-treatment increased the MDA level significantly in rat hippocampus compared with the other groups $\left({ }^{* * *} \mathrm{P}<0.001\right)$. MDA, malondialdehyde; Hcy, homocysteine.

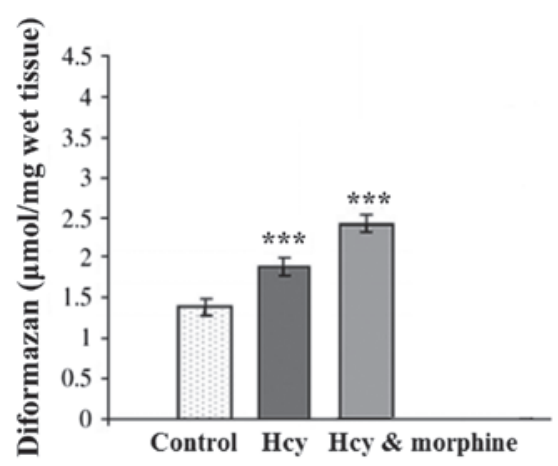

Figure 3. Effects of morphine (10 mg/kg) on SOA (Diformazan) concentration in Hcy $(0.5 \mu \mathrm{mol} / \mu \mathrm{l})$-treated rats. Morphine pre- and post-treatment increased SOA (Diformazan) significantly in rat hippocampus compared with the other groups $\left({ }^{* * *} \mathrm{P}<0.001\right)$. SOA, superoxide anion; Hcy, homocysteine.

Estimation of oxidative stress parameters. Fig. 2 shows the effects of drugs on lipid peroxidation. One-way ANOVA indicated that MDA level in the Hcy group was increased significantly compared to the sham group $(\mathrm{P}<0.001)$. Morphine pre and post treatment increased the MDA level significantly in rat hippocampus compared with the other groups $(\mathrm{P}<0.001)$.

Fig. 3 shows the effects of drugs on SOA level $(\mu \mathrm{mol} / \mathrm{mg}$ wet tissue) in the rat hippocampal homogenate. One-way 

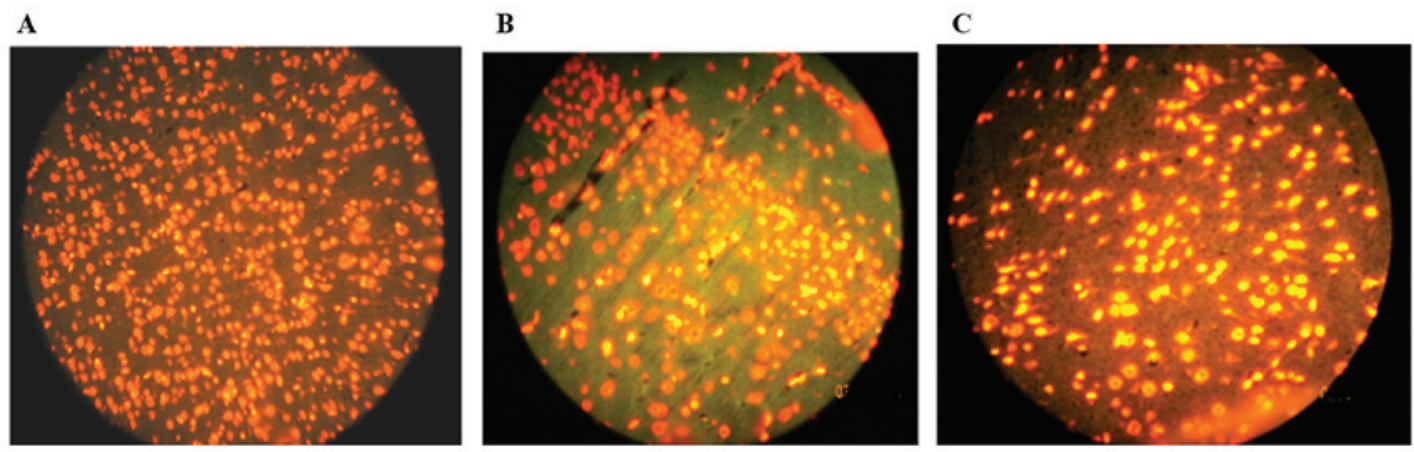

Figure 4. Effect of morphine $(10 \mathrm{mg} / \mathrm{kg})$ and $\mathrm{Hcy}(0.5 \mu \mathrm{mol} / \mu \mathrm{l})$ on apoptosis level (percentage of cell death) in rat hippocampus cells (P<0.001). TUNEL-positive cells in rat hippocampus: (A) sham group, (B) Hcy group, (C) Hcy-morphine using fluorescence microscopy Ex/Em wavelength $(\mathrm{Ex} / \mathrm{Em}=495 / 519 \mathrm{~nm})$. Hcy, homocysteine; TUNEL, transferase dUTP nick-end labeling.

ANOVA indicated that SOA level in the Hcy group was significantly greater than that of the sham group. In addition, morphine pre- and post-treatment led to a significant increase in the SOA level $(\mathrm{P}<0.001)$.

Evaluation of hippocampus cell apoptosis. As shown in Fig. 4, TUNEL staining was used to evaluate hippocampus cell apoptosis. It was found that Hcy could separately induce apoptosis in hippocampus cells and significantly increased the number of TUNEL-positive cells in rat hippocampus compared to the control group $(\mathrm{P}<0.001)$. Of note, our results indicated that morphine pre- and post-treatment did not decrease TUNEL-positive cells in rat hippocampus cells compared with the other groups (Fig. 5). Furthermore, pre- and post-treatment by morphine increased apoptosis in hippocampus cells compared with the other groups $(\mathrm{P}<0.001)$.

\section{Discussion}

Opioids as strong analgesics have been used in pain treatment for more than 100 years (13). Previous results suggested that morphine possesses some neuroprotective effects in different ischemia/reperfusion models (14). However, many investigations showed that morphine could protect against neuronal cell death, there are several lines of evidence suggest that morphine could induce apoptosis in neurons. Therefore, the current study was conducted to investigate the pre- and post-conditioning effects of morphine on hippocampal cell apoptosis in a rat model of Hcy-induced oxidative stress. Our results showed that Hcy separately induced apoptosis in hippocampus cells and significantly increased the number of TUNEL-positive cells in rat hippocampus compared to the control group. In addition, MDA and SOA levels in the Hcy group were increased significantly compared to the control group. It was in accordance with previous reports that the expression of apoptosis regulatory proteins, Bax and Bcl-2, would be altered by Hcy (15). It was also suggested that Hcy generates reactive oxygen species, which attacks the polyunsaturated fatty acids of neuronal cell membranes and induces lipid peroxidation in the hippocampus (16). Hcy is a non-protein amino acid, or thiol-containing amino, which is derived from methionine (17). Elevated plasma Hcy levels have been associated with high incidence of atherosclerotic and neurodegenerative disorders, such as Alzheimer's disease and dementia $(18,19)$. Evidence

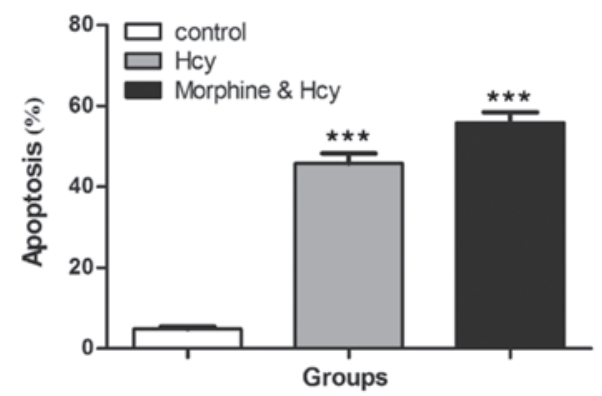

Figure 5. Comparison of apoptosis level (percentage of cell death) in rat hippocampus cells in the control, Hcy and Hcy-morphine groups $\left({ }^{* * *} \mathrm{P}<0.001\right)$ Hcy, homocysteine.

shows that Hcy is toxic to neuronal cells both in vitro and in vivo $(20,21)$ and can cause calcium influx, oxidative stress, neuroinflammation and neuronal apoptosis (22). Kruman et al also demonstrated that Hcy elicits a DNA damage response in rat hippocampal neurons that promotes apoptosis and hypersensitivity to excitotoxicity (21). In addition, Maler $\mathrm{et}$ al reported that Hcy induces cell death of rat astrocytes in vitro (23). Moreover, several animal models have shown a role of Hcy in cerebrovascular pathology, cognitive decline and learning disabilities (24). Additionally, in our previous reports, we demonstrated the neuroprotective effects of the polyphenolic antioxidant agent, Curcumin, against Hcy-induced cognitive impairment and oxidative stress in the rat (25). In previous reports by our research group, Hcy decreased locomotor activities significantly in rats as well as it could induce apoptosis in substantia nigra cells (26). Therefore, our previous experiences and use of Hcy in other published studies as animal models to induce oxidative stress, neuroinflammation and neuronal apoptosis can confirm the success of the model. Moreover, the present study was substantially revised and developed by further experiments such as analysis of structure and morphology of hippocampal cells by H\&E and assay of MDA and SOA in hippocampal homogenate which can approve the success of the model.

Our results have demonstrated that morphine pre- and post-treatment could not decrease TUNEL-positive cells in rat hippocampus cells. On the other hand, pre- and post-treatment by morphine increased apoptosis in hippocampus cells compared with the other group. Contrast to our results, previous studies suggested neuroprotective effects of morphine against 
neuronal cell death. In rat neuronal/glial cultures, morphine was reported to prevent cell death induced by HIV envelope glycoprotein gp120IIIB or BaL (5). In addition, Zhao et al reported that morphine pre-conditioning induces opioid receptor-dependent neuroprotection against ischemia in rats and reduces ischemia-induced cell death in the CA1 regions of hippocampal slices (2). Several lines of evidence indicated that the induction of estradiol release in hippocampal neurons by morphine induces upregulation of heat shock protein 70 that protects against neuronal damage and cell death (27). Despite these facts, several other investigations confirmed our results. Liu et al stated that long-term use of morphine can induce neuronal apoptosis in the brain by increasing the expressions of pro-apoptotic Fas and caspase- 3 and decreasing the anti-apoptotic Bcl-2 expression as an underlying mechanism for the opiate-induced neuronal damage (8). Moreover, in an investigation regarding the effect of morphine on apoptosis in the nucleus accumbens in rat brain, the results showed that apoptotic factors increased in all the groups treated with morphine (28). It can be hypothesized that these apoptotic effects may be attributed to effects of opioids on neuronal structure (cytoskeleton), which can lead to neuronal damage.

Taken together, our data suggested that morphine pre- and post-conditioning exacerbates apoptosis and oxidative stress in hippocampus cells. Although this study achieved its aims, there were some unavoidable limitations. First, because the neuroprotective or neurotoxic effect of morphine may be related to dose, a dose-escalating treatment is suggested to be performed in future studies. Second, future investigations should consider a protective or neurotoxic effect of morphine through analysis of the expression of pro- and anti-apoptotic genes on mRNA and/or protein level and metabolism genes. Finally, since improved understanding of morphine neuroprotection and neurotoxicity is useful to control morphine side-effects in medical applications and to identify new targets for potential therapies and prevention strategies to opioid addiction, further studies are needed to reveal the exact mechanism of morphine in cell death process (apoptosis) or the neuroprotective properties of morphine should be studied in more detail.

\section{Acknowledgements}

The authors would like to thank Dr Azadmehr, Dr Zabihi, and Dr Ali Akbar Moghadamnia for their assistance and effective comments. This study was supported by the Cellular and Molecular Biology Research Center, Babol University of Medical Sciences, Babol, Iran.

\section{References}

1. Zhou Q, Krebs JF, Snipas SJ, Price A, Alnemri ES, Tomaselli KJ and Salvesen GS: Interaction of the baculovirus anti-apoptotic protein p35 with caspases. Specificity, kinetics, and characterization of the caspase/p35 complex. Biochemistry 37: $10757-10765,1998$.

2. Zhao P, Huang Y and Zuo Z: Opioid preconditioning induces opioid receptor-dependent delayed neuroprotection against ischemia in rats. J Neuropathol Exp Neurol 65: 945-952, 2006.

3. Sanchez-Simon FM, Arenzana FJ and Rodriguez RE: In vivo effects of morphine on neuronal fate and opioid receptor expression in zebrafish embryos. Eur J Neurosci 32: 550-559, 2010.
4. Qian L, Tan KS, Wei SJ, Wu HM, Xu Z, Wilson B, Lu RB, Hong JS and Flood PM: Microglia-mediated neurotoxicity is inhibited by morphine through an opioid receptor-independent reduction of NADPH oxidase activity. J Immunol 179: 1198-1209, 2007.

5. Avdoshina V, Biggio F, Palchik G, Campbell LA and Mocchetti I: Morphine induces the release of CCL5 from astrocytes: Potential neuroprotective mechanism against the HIV protein gp120. Glia 58: 1630-1639, 2010.

6. Ammon-Treiber S, Stolze D, Schröder H, Loh H and Höllt V: Effects of opioid antagonists and morphine in a hippocampal hypoxia/hypoglycemia model. Neuropharmacology 49: 1160-1169, 2005.

7. Liu Y, Nie YM and Wu WK: The effect of PKC activation and Smac release on inhibition of myocardial cell apoptosis by Sini Decoction. Zhong Yao Cai 31: 1675-1678, 2008 (In Chinese).

8. Liu LW, Lu J, Wang XH, Fu SK, Li Q and Lin FQ: Neuronal apoptosis in morphine addiction and its molecular mechanism. Int J Clin Exp Med 6: 540-545, 2013.

9. Mao J, Sung B, Ji RR and Lim G: Neuronal apoptosis associated with morphine tolerance: Evidence for an opioid-induced neurotoxic mechanism. J Neurosci 22: 7650-7661, 2002.

10. Yin D, Mufson RA, Wang R and Shi Y: Fas-mediated cell death promoted by opioids. Nature 397: 218, 1999.

11. Placer ZA, Cushman LL and Johnson BC: Estimation of product of lipid peroxidation (malonyl dialdehyde) in biochemical systems. Anal Biochem 16: 359-364, 1966.

12. Das UN, Padma M, Sagar PS, Ramesh G and Koratkar R: Stimulation of free radical generation in human leukocytes by various agents including tumor necrosis factor is a calmodulin dependent process. Biochem Biophys Res Commun 167: 1030-1036, 1990.

13. Barry U and Zuo Z: Opioids: Old drugs for potential new applications. Curr Pharm Des 11: 1343-1350, 2005.

14. Lim YJ, Zheng S and Zuo Z: Morphine preconditions Purkinje cells against cell death under in vitro simulated ischemia-reperfusion conditions. Anesthesiology 100: 562-568, 2004.

15. Ataie A, Ataee R, Shadifar M, Shahabi S, Aghajanpour SM and Hosseinpour Y: Interaction of Memantine with homocysteine on the apoptosis in the rat hippocampus cells. Int J Mol Cell Med 1: $145-152,2012$.

16. Agnati LF, Genedani S, Rasio G, Galantucci M, Saltini S, Filaferro M, Franco R, Mora F, Ferré S and Fuxe K: Studies on homocysteine plasma levels in Alzheimer's patients. Relevance for neurodegeneration. J Neural Transm (Vienna) 112: 163-169, 2005.

17. Selhub J: Homocysteine metabolism. Annu Rev Nutr 19: 217-246, 1999.

18. Biasioli S and Schiavon R: Homocysteine as a cardiovascular risk factor. Blood Purif 18: 177-182, 2000.

19. Sachdev P: Homocysteine, cerebrovascular disease and brain atrophy. J Neurol Sci 226: 25-29, 2004.

20. Lipton SA, Kim WK, Choi YB, Kumar S, D'Emilia DM, Rayudu PV, Arnelle DR and Stamler JS: Neurotoxicity associated with dual actions of homocysteine at the N-methyl-D-aspartate receptor. Proc Natl Acad Sci USA 94: 5923-5928, 1997.

21. Kruman II, Culmsee C, Chan SL, Kruman Y, Guo Z, Penix L and Mattson MP: Homocysteine elicits a DNA damage response in neurons that promotes apoptosis and hypersensitivity to excitotoxicity. J Neurosci 20: 6920-6926, 2000.

22. Obeid R and Herrmann W: Mechanisms of homocysteine neurotoxicity in neurodegenerative diseases with special reference to dementia. FEBS Lett 580: 2994-3005, 2006.

23. Maler JM, Seifert W, Hüther G, Wiltfang J, Rüther E, Kornhuber J and Bleich S: Homocysteine induces cell death of rat astrocytes in vitro. Neurosci Lett 347: 85-88, 2003

24. Kamat PK, Vacek JC, Kalani A and Tyagi N: Homocysteine induced cerebrovascular dysfunction: A link to Alzheimer's disease etiology. Open Neurol J 9: 9-14, 2015.

25. Ataie A, Sabetkasaei M, Haghparast A, Moghaddam AH and Kazeminejad B: Neuroprotective effects of the polyphenolic antioxidant agent, Curcumin, against homocysteine-induced cognitive impairment and oxidative stress in the rat. Pharmacol Biochem Behav 96: 378-385, 2010.

26. Ataie A, Ataee R, Mansoury Z and Aghajanpour M: Homocysteine intracerebroventricular injection induces apoptosis in the substantia nigra cells and Parkinson's disease likebehavior in rats. Int J Mol Cell Med 2: 80-85, 2013.

27. Cui J, Wang Y, Dong Q, Wu S, Xiao X, Hu J, Chai Z and Zhang Y: Morphine protects against intracellular amyloid toxicity by inducing estradiol release and upregulation of Hsp70. J Neurosci 31: 16227-16240, 2011.

28. Katebi N, Razavi Y,Zeighamy Alamdary S, Irani S, Khodagholi F and Haghparast A: Effect of morphine on apoptotic factors caspase-3, PARP and Bax/Bcl-2 ratio in nucleus accumbens in conditioned place preference model in rat. Physiol Pharmacol 17: 39-50, 2013. 